\title{
marges Marges
}

revue d'art contemporain Revue d'art contemporain

$10 \mid 2010$

Déplacements des pratiques artistiques

\section{Cinéma et art contemporain, nouvelles approches de l'essai}

Véronique Terrier Hermann

\section{OpenEdition}

\section{Journals}

Édition électronique

URL : http://journals.openedition.org/marges/499

DOI : $10.4000 /$ marges.499

ISSN : 2416-8742

\section{Éditeur}

Presses universitaires de Vincennes

\section{Édition imprimée}

Date de publication : 15 avril 2010

Pagination : 86-100

ISBN : 978-2-84292-254-2

ISSN : $1767-7114$

\section{Référence électronique}

Véronique Terrier Hermann, «Cinéma et art contemporain, nouvelles approches de l'essai », Marges [En ligne], 10 | 2010, mis en ligne le 15 avril 2010, consulté le 19 avril 2019. URL : http:// journals.openedition.org/marges/499; DOI : 10.4000/marges.499 


\section{Cinéma et art contemporain, nouvelles approches de l'essai.}

/1 Jean-Luc Godard, Histoire(s) du cinéma (1989-1998).

/ 2 Ces titres sont empruntés à ceux de manifestations récentes dont les rencontres «Où va le cinéma?", organisées par Les Inrockuptibles et le Centre Pompidou en décembre 2008, tentaient de présenter l'ensemble des directions actuelles du cinéma.

/3 Sous l'impulsion de Christian Merlhiot, Erik Bullot, Vincent Dieutre, Pascale Cassagnau, en partenariat avec le GREC, la DAP, et la Fémis. Voir l'ouvrage d'Érik Bullot (sld), Pointligneplan, Paris, Léo Scheer, 2002.

/4 Maison de production de films en partenariat avec le Consortium, qui a produit notamment
"Avec Édouard Manet commence la peinture moderne. C'est-à-dire le cinématographe. C'est à dire des formes qui cheminent vers la parole. Très exactement une forme qui pense /1. »

«Où va le cinéma?», "Réel et fiction, un retour de la question», "Cinéma en numérique », «De la salle au musée /2 »... L'actualité est riche en cycles de programmation, publications, colloques ou tables rondes qui, tous à leur manière, se font l'écho d'une émergence de pratiques cinématographiques différentes. Autant de questions et de tentatives pour définir ce qui se joue, de manière chaque jour plus manifeste, dans le champ rapproché de l'art contemporain, du documentaire et du cinéma. Car au-delà des glissements de toujours entre art et cinéma, on assiste dernièrement à un véritable débordement du cinéma; phénomène qui se propage depuis une dizaine d'années, grâce notamment à la plus grande latitude donnée au cinéma par la vidéo numérique. En France, c'est en 1998 que l'association Pointligneplan/3, présentée au croisement du cinéma et de l'art contemporain, initie la diffusion régulière, en salle, de films réalisés par des artistes. Elle sera naturellement suivie par bien d'autres structures, institutions et manifestations (Anna Sanders Films /4, Centre Georges Pompidou, Jeu de Paume, Festival International du Documentaire, etc.). Sur la scène internationale de l'art, on ne mentionnera que les Documenta 10 et $11 / 5$ comme des manifestes d'une large ouverture au film, mais nombreux sont les témoins privilégiés de cette porosité 
entre art contemporain et cinéma. Tous, artistes et critiques, s'accordent pour constater qu'un nouveau territoire s'est dessiné et revendique sa différence, que l'ancienne division des genres n'est plus tenable aujourd'hui. Reste alors - dans une volonté de se démarquer des termes de "Cinéma expérimental», d' "art vidéo» ou de «films d'artistes»-, la difficulté de trouver une terminologie, une articulation. Plusieurs propositions émergent, allant «D’un autre cinéma /6 » de Raymond Bellour, au «Tiers-cinéma /7» de Vincent Dieutre ou au «Troisième cinéma/8 » de Pascale Cassagnau. Toutefois, combien de films à la marge de l'art ou du cinéma échappent-ils encore à la critique, aux tentatives de définitions, aux classements par genre ou par champ ? Combien sont-ils à être présentés dans un «entre-deux», entre cinéma et art contemporain, entre fiction et documentaire, entre cinéma expérimental et vidéo?

Quoique qualifiées d'«Fuvres singulières inquiétant les lignes de partage entre documentaire et fiction, journal filmé et essai, fonction narrative et expérimentation plastique, cinéma et art contemporain/9", elles occupent pourtant pleinement un territoire, investi par des auteurs que l'on trouve tout autant du côté des expositions que des festivals de cinéma. Citons Dominique Gonzalez-Foerster, Clarisse Hahn, Valérie Jouve, Ariane Michel, Valérie Mréjen, Philippe Parreno, Anri Sala, Olivier Zabat/10... Autant de noms comme autant de différences de styles, de formes et d'expériences qui s'affirment au sein de ce territoire composite. C'est pourquoi il nous faut souligner ce qui, dans cette pluralité, s'articule comme dénominateur commun, partage une même volonté. C'est en se tournant vers le rapport entre le cinéma et le genre de l'essai que l'on tentera de trouver ces articulations communes. Genre inclassable et nomade, l'essai au cinéma rassemble des «œuvres-limites qui transgressent la logique de séparation des genres et/ou radicalisent une forme d'écriture et notamment celle du documentaire/11». Aussi, l'essai peut-il nous inviter à reconsidérer les films réalisés par des artistes contemporains, non plus dans cet "entre-deux» de l'art et du cinéma, de la fiction et du documentaire, mais à l'aune du cinématographique. D’où notre proposition : révéler les correspondances entre film-essai, film de l'art contemporain et faire glisser le territoire encore indéfini de ces films vers celui du cinéma.

\section{De l'essai littéraire à l'essai cinématographique}

Bien que l'histoire de l'essai trouve sa source chez Montaigne, de nombreux auteurs enrichissent le corpus de cette forme littéraire jusqu'au vingtième siècle/12. Figure ouverte et réflexive, l'essai est les films de GonzalezFoerster, Huyghe, Parreno, de Meaux et Weerasethakul. Voir The In-Between. Anna sanders Films, Dijon, Forma/Les Presses du Réel, 2003, compilation critique des films produits par Anna Sanders.

/5 Catherine David (sld), Documenta 10 Politicspoetics, Ostfildern-Ruit, Hatje Cantz, 1997, et Okwui Enzvenor (sld), Documenta 11_Platform 5 , Ostfildern-Ruit, Hatje Cantz, 2002.

/6 Raymond Bellour, "D’un autre cinéma », Trafic, $\mathrm{n}^{\circ} 34,2000$.

/7 Vincent Dieutre, «Le Tiers-Cinéma est proclamé, vive le Tiers-Cinéma!», La Lettre du cinéma, $n^{\circ} 21$, «Abécédaire du Tiers-Cinéma », Hiver 2003.

/8 Pascale Cassagnau, Future Amnesia, enquêtes sur un troisième cinéma, Paris, Isthme éditions, 2007.

/ 9 Erik Bullot, présentation de la collection pointligneplan en vod, mars 2009.

/10 Cette liste renvoie aux artistes sur lesquels est basé cet article, mais nous pourrions la prolonger en citant Kutlug Ataman, Emmanuelle Antille, Ursula Biemann, Harun Farocki, Walid Raad, Allan Sekula, Gillian Wearing, et bien d'autres encore. 
/11 José Moure, «Essai de définition de l'essai au cinéma », dans Suzanne Liandrat-Guigues et Murielle Gagnebin (sld), L'Essai et le cinéma, Paris, Champ Vallon, 2004, p. 27.

/12 Voir à ce sujet Marielle Macé, Le Temps de l'essai. Histoire d'un genre en France au XXe siècle, Paris, Belin, 2006 et Claire de Obaldia, L'Esprit de l'essai, De Montaigne à Borges, Paris, Seuil, 2005.

/13 Jean Starobinski, «Peut-on définir l'essai?», Cahiers pour un temps, Paris, Centre Georges Pompidou, 1985.

/14 Voir Suzanne Liandrat-Guigues, "Un art de l'équilibre», dans L'Essai et le cinéma, op. cit., p. 8.

/15 ibid., p. 8-9.

/16 Noël Burch, Une Praxis du cinéma, Paris, Gallimard, 1969, p. 227.

/17 André Bazin, "Deux documentaires », Radio-Cinéma, $\mathrm{n}^{\circ} 461$, 16 novembre 1958 , cité par Arnaud Lambert, Also known as Chris Marker, Cherbourg, Le Point du Jour, 2008, p. 61. avant tout une pratique de la pensée. Composé d'une pluralité d'approches allant de la littérature à la philosophie et même aux sciences, l'essai se cherche encore. Starobinski relance d'ailleurs la question de sa définition - «Peut-on définir l'essai /13 ? »-, pour finalement faire le constat que l'essai ne se soumet à aucune règle. Face à cette réticence à toute définition, seules sont alors pensables ses conditions d'apparition. Essayons pourtant d'avancer à partir de ses caractéristiques: l'essai est un texte ouvert qui se prête à la réflexion. Parcourant le réel, il transcrit son expérience - non dans l'établissement d'un savoir -, mais dans une dimension sensible de la pensée. Libre de règles et de contraintes, il se construit dans une esthétique qui ne cesse de se renouveler.

Objet littéraire ainsi présenté comme non définissable, comment l'appréhender au cinéma? Le glissement s'opère à partir de son sens étymologique: une pesée, une balance. Aussi, le cinéma de l'essai serait un cinéma de l'équilibre, un cinéma toujours sur la brèche, qui prendrait le risque de s'expérimenter, de se mettre à l'épreuve/14. Son acceptation dans le champ du cinéma revêt d'ailleurs plusieurs formes, des avant-gardes à nos jours. "Les premières manifestations de l'emploi du mot essai, dans les années 1920, chez Eisenstein ou en 1940, chez Hans Richter, ne recouvrent pas exactement ses réactualisations dans les années 1960, en relation avec ce que la critique appellera la modernité. Une fois dégagée des implications narratives qui ont fait naître le constat de modernité [...], celle-ci ne se définit plus guère sinon par l'usage du terme essai appliqué aux films les plus divers - et d'abord à des films documentaires au sens que ce mot revêt dans les œuvres d'un Franju, d'un Resnais, d'une Varda ou d'un Marker /15. »

Bien que Georges Franju dans Hôtel des Invalides (1951) conserve encore des caractéristiques propres au documentaire et à la fiction (unité des matériaux, de ton et de style), Noël Burch en fait tout de même l'initiateur des «films-en-forme-d'essai». "Ces non-fictions qui n'étaient pas des documentaires dans le sens objectif: c'étaient des films dont le propos était d'exposer des partis-pris et des contre-partis à travers la facture même du film; leur forme était une méditation, leurs sujets étaient des conflits d'idées contenus dans le thème; et, fait capital, de ces conflits sont nées des structures /16.» Bazin, à son tour, adopte l'essai afin de parler de Lettres de Sibérie de Chris Marker, tout en précisant: "un essai documenté par le cinéma. Le mot important n'étant pas cinéma mais essai entendu non dans le sens d'expérience ou de tentative mais dans l'acception littéraire /17. » Référence de la Modernité, le terme regroupe alors des films qui tissent encore des liens avec le documentaire, mais opèrent 
en même temps la conversion des signes cinématographiques vers des formes inédites. Sans opérer de détour par le cinéma expérimental, le film-essai est dès lors reconnu tant par la critique que par les cinéastes et se joue principalement sur le diapason de l'inventivité et de la force d'indépendance de la pensée.

\section{L'essai comme forme entre documentaire et fiction}

\section{"Le réel doit être fictionné pour être pensé /18 "}

Ces derniers temps, c'est la porosité entre fiction et documentaire qui se manifeste de manière la plus évidente. Du côté du cinéma, Emmanuel Burdeau s'en fait le messager, «il y aura dans les mois, dans les années à venir, de plus en plus de films à faire avec: la fiction avec le documentaire, le documentaire avec la fiction. Mille éléments y incitent, la libération qu'a été et qu'est encore le grand débordement des pratiques documentaires; la multiplicité des images et de leurs régimes, de leurs provenances, de leurs circulations; les frottements toujours plus nombreux entre l'art et le cinéma ; la légèreté technique du numérique; [...] II n'y a pas une voie, mais autant de voies que de films cherchant dans l'articulation du documentaire et de la fiction de nouveaux accords, de nouvelles traductibilités /19. »

Toutefois, si l'ensemble des films dont nous traitons associe documentaire et fiction, ce n'est pas tant dans une quête de leurs similarités ou de leurs différences, que dans une volonté d'ouvrir un espace, de créer un lieu propice à ce métissage réflexif. "Le film-essai se caractérise en outre par le fait de s'installer dans une zone d'indétermination entre la non-fiction et la fiction. [...] Le film-essai assume cette indétermination, la travaille, la réfléchit, en fait l'objet même de sa méditation /20 ». Indétermination assumée, le sujet peut être abordé de manière tout à fait personnelle, poétique, voire méditative.

Repensons au film Ruins of Love (2005) de Ange Leccia où la beauté du film est valorisée par la musique, telle une ballade musicale, un clip. Toutefois les images sont celles de prostituées cambodgiennes qui apparaissent, par un cadrage précis, comme cloisonnées dans leur destin, coincées entre les barbelés d'enceinte d'un ancien camp khmer rouge où le passage des phares des voitures évoque le balayage lumineux des enceintes des prisons. Les images du film, ainsi suspendues et cloisonnées, pourraient convoquer l'espace étroit du temps quand il est pris en étau entre le passé et le présent, - métaphore de l'histoire cambodgienne?

L'apport de la fiction est un choix délibéré, une manière de condenser du sens, d'interroger la réalité jusqu'à nous rappeler que la vérité ne loge pas exclusivement dans le réel. L'essai fonctionne comme
/18 Jacques Rancière, Le Partage du sensible, Paris, La Fabrique, 2000, p. 61.

/19 Emmanuel Burdeau, «Be with me, la fiction avec le documentaire», Marseille, FID, 2006.

/ 20 José Moure, "Essai de définition de l'essai au cinéma», op. cit., p. 36-37. 
/21 François Niney, L'Épreuve du réel à l'écran, Bruxelles, De Boeck, 2002, p. 297.

/22 José Moure, "Essai de définition de l'essai au cinéma», op. cit., p. 36. réflexion sur le monde à partir d'une remise en forme du réel. C'est d'ailleurs ce que suggère François Niney au sujet d'une pléiade de documentaristes tels Robert Kramer, Johan van der Keuken ou même Harun Farocki, qui relèvent le défi du réel. "En réveillant les puissances positives et déstabilisantes de l'illusion (désillusion), du (dé) trompe-l'œil, de l'incertitude (où s'arrête la fiction, où commence le réel?), ces cinéastes remettent en jeu notre présence et notre croyance au monde, la vérité du réel. La fiction vient subvertir l'ordre apparent de la réalité, le remettre en cause; le réel vient couper dans la fiction, contestant sa vraisemblance /21. ».

Pour Voyage au pays des vampires (2001) de Christian Merlhiot, la caméra prend la place d'un des participants de ce voyage organisé en Transylvanie. Le réel y prend place, mais sans faire effet. On y suit alors le trajet annoncé, qui chemine entre réalité des lieux et fantasme colporté par les nombreuses histoires littéraires et cinématographiques. Dans une constante expérimentation, tout est maîtrisé par la mise en scène du réel qui réinstalle, par le montage, les possibles fictionnels.

Puisque l'essai opère entre fiction et non-fiction, quel est donc ce processus mis en œuvre qui le distingue du documentaire dont il est pourtant si proche? Selon Moure, le film-essai ne part pas de la réalité, mais est construit comme un langage, par élaboration de signes sonores et visuels, avec, de surcroît, une réflexion sur ses conditions d'apparition. "Le film essai semble en effet fortement apparenté au documentaire. [...] Mais alors que dans le documentaire, cette réflexion repose sur le présupposé d'un rapport presque naturel à une réalité donnée, dans le film-essai, le travail filmique se produit non à partir d'une réalité, mais à partir de matériaux sonores et visuels dont la structuration ou combinaison non seulement laisse visibles les traces d'un processus de pensée, mais les incorpore à la texture même du film et joue sur leurs tensions /22. ».

Le travail de Valérie Jouve est caractéristique de cette radicalisation du documentaire. En parallèle à un travail photographique basé sur les territoires urbains dont elle revendique le style documentaire, elle s'est engagée dans une œuvre cinématographique qui puise dans le réel et la fiction. Son premier film, Grand Littoral (2003) suit les déambulations d'une communauté fragile d'individus sur une colline de Marseille, coincée entre habitat HLM, réseau routier et centre commercial. Tentant de venir à bout de la relation qu'elle entretient depuis longtemps avec la question de l'occupation possible d'un territoire, elle élabore par la mise en scène et le montage, une sorte de chorégraphie urbaine de passages et de rencontres. Composé d'une pluralité d'approches visuelles, la caméra de Munster Lands 
(2007) suit le parcours de chacun des personnages selon leurs modes d'arrivée (rail, route, voie d'eau), comme autant de découvertes de la ville qui se recomposent dans le film. Travaillant aussi les éléments du film comme un collage, Jouve a assemblé Time is working around Rotterdam (2006) de manière à former une composition visuelle de rythmes. Tout y est concentré sur la vitesse, ou plutôt, sur l'ensemble des vitesses qui scandent toute une ville en activité. Les passages du centre à la périphérie, les nombreux travellings horizontaux, mais aussi les échappées, les trains en mouvement, les déambulations des piétons, la musique spécialement composée, le montage. Tout concourre à mettre en symphonie le travail du temps. La caméra de Jouve, à l'écoute des sons, rend ces flux et rythmes dans une saillance du réel, afin de montrer que la ville ne dicte pas une seule temporalité, bien au contraire, elle se construit et s'alimente de cette diversité des vitesses, des concordances ou même des ruptures de temps.

Lorsque Jacques Rancière élabore son concept d'histoire poétique, il repousse plus loin le binôme fiction/réel de l'essai, pour en faire une nécessité. "Cette articulation est passée de la littérature au nouvel art du récit, le cinéma. Celui-ci porte à sa plus haute puissance la double ressource de l'impression muette qui parle et du montage qui calcule les puissances de signifiance et les valeurs de vérité. Et le cinéma documentaire, le cinéma voué au réel est, en ce sens, capable d'une invention fictionnelle plus forte que le cinéma de fiction /23.» Et quand il avance que "le réel doit être fictionné pour être pensé », c'est après avoir choisi comme exemple Le Tombeau d'Alexandre (1993) de Chris Marker, à savoir, un film-essai qui questionne le travail de falsification des images, de représentation du réel. Le film traite de la représentation de la révolution soviétique à travers le travail du cinéaste Alexandre Medvedkine. Par la mise à plat de la fabrique des images, ce film est une relecture, non de l'Histoire, mais d'une vision de l'Histoire.

The Battle of Orgreave (2001) de Mike Figgis et Jeremy Deller a pour sujet un événement d'Histoire: un affrontement historique entre mineurs et forces de l'ordre qui signe la fin de l'industrie minière en Grande-Bretagne, mais aussi son corollaire, l'épuisement du pouvoir des syndicats. Le film procède de deux registres, la scène rejouée de l'affrontement et une série d'entretiens des acteurs de l'époque. Le propos dépasse la simple analyse de ce drame. Le document y prend son relief à l'aune du montage dans lequel il se déploie - et qui permet de mettre de l'ordre, de mettre en mémoire. Aussi, par cette proximité de différents modes de pensée et de représentation entre fiction et réel, il interroge tout travail de réflexion politique qui se base sur des images.
/ 23 Jacques Rancière, Le Partage du sensible, op. cit., p. 60. 
/24 Murielle Gagnebin, op. cit., p. 17.

/25 Pascale Cassagnau, op. cit., p. 12.

/26 Stéphanie Moisdon, «m.m.m. (moments, mondes, modernités)», dans Pointligneplan, op. cit, p. 106.

/ 27 Voir Geneviève Loup, «Anna Sanders comme dispositif critique de la position de l'auteur dans l'œuvre de Pierre Huyghe", Décadrages, n¹3, 2008.

/ 28 The In-Between. Anna Sanders Films, op. cit., dernière de couverture.
N'ayant pas vocation à rester neutre, le film-essai - contrairement au documentaire - peut-être partisan, polémique et s'affirme comme prise de parole assumée. Croisant la fiction et le documentaire dans une reformulation du réel, il est avant tout une réflexion par l'image.

\section{L'essai comme forme qui se risque}

Pour le film-essai, faire le choix d'un sujet c'est faire le choix d'une forme esthétique. Mais l'essai se reconnaît aussi par ses non-dits, «son contingent de fantaisies irrésolues, tant verbales qu'iconiques (choix des plans, des mouvements de caméra, des raccords audacieux), ou de fantaisies afférentes aux multiples formes de montage [...]. L'inconscient de l'auteur se pliant en quelque sorte devant l'inconscient de l'œuvre elle-même /24 ».

De plus, l'essai comme forme qui se risque implique un récit d'expériences cinématographiques. Concordance majeure du film-essai avec les productions d'art contemporain, la réflexion autour du dispositif à l'œuvre manifeste sa présence dans la forme finale. "Ces univers cinématographiques sont autant d'expériences de récits que de récits d'expériences, qui portent le cinéma dans l'espace de l'essai/25. ». Écrivant sur les films de Dominique Gonzalez-Foerster, Stéphanie Moisdon choisit de souligner ce qui tient du processus, du récit d'expériences autour de la création même du film, tant il persiste dans la forme. "C'est un film-théorie sur le récit vécu d'un dispositif filmique /26. ». Cinéma réflexif / 27, prégnance du dispositif, alternance des références, l'essai tire sa forme d'une somme d'expériences, d'expérimentations, de perturbations, de prises de risque, d'allers et retours. C'est d'ailleurs ainsi que se définit Anna Sanders Films, "quelque part dans cet entre-deux: entre deux économies, entre deux rêves, entre deux réalités, entre deux fictions, entre deux cinémas /28 ».

Cette réflexion sur la forme amène donc les artistes à élaborer des dispositifs réflexifs, tant du côté de la fiction que de celui du documentaire. Ainsi, quand le film se joue sur le mode de la fiction, il semble notamment inviter le cinéma comme éléments de citation ou d'articulation. Non ricordo il titolo (J'ai oublié le titre) (2008) de Christelle Lheureux met en scène les sosies de Marcello Mastroianni et Ingrid Bergman sur les pentes du volcan de Stromboli. Autant d'indices cinématographiques accentués par l'insistance du montage et des scènes répétées qui ont pour effet de convoquer notre mémoire. Allusion au cinéma, la remembrance opère, mais un souvenir en chasse un autre et contrarie finalement le phénomène de rémanence ainsi convoqué, qui ne peut rassembler nos souvenirs - de fait, ces deux acteurs ne se 
sont jamais rencontrés dans un film de Rossellini, de Fellini ni même d'Antonioni. Mais forte de l'effet cinéma, l'expérience a eu prise sur notre mémoire.

D'autre part, quand le film se joue sur le mode du documentaire, c'est aussi une forme qui intègre son mode de réflexion même. Pour exemple, les témoignages ostensiblement dirigés de Valérie Mréjen dans Portraits filmés (2002) ou Chamonix (2002), énoncent avec une certaine dérision des souvenirs inspirés d'événements quotidiens, de lieux communs ou de malentendus. S'ils se distinguent du documentaire, c'est à leur manière qu'ils ont de mettre en évidence la réécriture, la mise en scène, la présence de la caméra. Cette prégnance du processus laisse les personnages comme coupés du monde, exclus de leur propre langage, révélant alors une certaine vacuité. Le réel est encore là, mais déjà dépossédé.

Ailleurs, c'est le montage réflexif de documents divers (de l'Histoire et des histoires) qui produit le récit, pouvant glisser vers la fiction. Les films d'Erik Bullot comme L'Attraction universelle (2000) ou Le Singe de la lumière (2002) exposent, à leur façon encyclopédique, des sons, des actions, des images. Le montage féconde les documents d'archive, croise les démonstrations mises en scène. Toutefois, loin de vouloir exposer un savoir, le film suit le déroulement d'une pensée, emprunte la forme de la ritournelle et joue plaisamment avec des savoirs.

Si la narration ou le récit n'est pas exclu, la mise en scène, semble elle, s'effacer petit à petit de ces films. Cela n'engendre pas pour autant un retour du réel, une image captée de la réalité à l'état brut, mais une recherche sur le processus. Lorsque Charles de Meaux présente son dernier film Marfa Mystery Lights, A Concert for the UFO's, 2006, il le compare à un process. Il sous-entend par là, non pas que son film est en cours, mais que ce qui s'y passe est quelque chose qui se joue précisément sur le mode du processus. A savoir, un groupe de musiciens se retrouve à Marfa pour écrire la musique de leur prochain concert, mais leur travail est troublé par le doute, la difficulté et surtout l'aspect chargé du lieu - croisement d'extra-terrestres et d'art minimal. Le film, qui suit ce processus de création du groupe, révèle aussi le processus du film en train de se chercher, de se découvrir.

L'essai comme forme qui se risque peut aller jusqu'à mettre en jeu sa relation au spectateur. "L'essai au cinéma implique, on le comprend, des échanges bien particuliers avec le spectateur, le contraignant tantôt à penser nouvellement, tantôt à conduire diverses transformations scéniques [...], ou encore le forçant à quelque disputatio, portant en creux la nécessité de mettre en crise la proposition insolente et insolite qu'est, au cinéma, l'essai /29. » Zidane, Un portrait du $21^{e}$ siècle (2006) de Philippe Parreno et Douglas Gordon, loin de
/29 Murielle Gagnebin, op. cit., p.21. 
/30 Ariane Michel, «Synopsis », [en ligne] http://arianemichel. monsite.wanadoo.fr/ page3.html

/31 «En un sens, D.G.F conçoit le cinéma comme une déambulation au sein d'un environnement où le naturel et l'artifice se confrontent, constituant ainsi un texte filmique condensé. » François Bovier et Geneviève Loup, «Indifférenciation et singularité dans les courts métrages de Dominique GonzalezFoerster ", Décadrages, op. cit., p. 41. rechercher un effet de réel du football, nous a permis de participer à une expérience cinématographique presque surréelle. Tout se joue dans le dispositif, qui consiste à cerner le sujet dans une proximité maximale par la mise en place sur le terrain d'un nombre impressionnant de caméras (17) toutes dirigées sur Zidane. Le film, qui rend le jeu quasiment abstrait, se focalise - dans un certain réalisme - sur un corps, extrait une figure, sujet unique, vecteur d'expérience.

Autre proposition insolite, Ariane Michel bouleverse ces échanges avec les spectateurs en les conviant dans un bois pour une mise en scène de la projection de son film The Screening (2007). Elle la présente de la manière suivante: "Synopsis: Une forêt, la nuit. Les rituels des bêtes nocturnes sont perturbés par la venue d'un groupe d'humains: ils s'assoient devant une surface blanche qui, plongée dans le noir, s'anime /30. ». Ici, c'est l'irruption même des spectateurs qui produit une mise en abyme du film puisqu'on y voit les effets de l'arrivée des hommes sur les animaux du bois. Relation de co-présence entre l'homme et l'animal, entre le film et sa projection, The Screening est un récit d'une expérience simultanée qui perturbe sciemment le rapport au regardeur.

\section{L'essai comme carnet de route}

Montaigne déclarait: "Je suis moi-même la matière de mon livre». Cinéma à la première personne, la matière est ici, non seulement quête de soi, mais aussi ensemble d'expériences, de rencontres, de territoires parcourus par l'auteur. Ainsi, différemment du documentaire qui se propose comme étude, l'essai est une quête personnelle, une découverte de soi et du monde; collecte vagabonde, cette quête peut devenir errance, image nomade.

Dans la compilation Parc Central (1998-2003) de Dominique GonzalezFoerster, on découvre des villes au cours de longs travellings, flâneries dans des parcs, dérives urbaines. Le fil ténu du film se joue entre linéarités d'images qui regardent et dérives de l'image qui «médite». Car ces portraits sensibles et méditatifs de lieux questionnent non pas tant l'artificialité de ces paysages que la réalité même de ces artifices, un cinéma comme déambulation entre réel et artefact/31. En arrivant à Hong Kong, l'artiste confie vouloir y rester pour toujours, «disparaître ici », « devenir Hong Kong ». S'immergeant dans le paysage jusqu'à vouloir faire corps avec lui, la caméra dévoile un regard qui se construit tout en se révélant et réveille en chacun une conscience de la ville. Conscience de la ville mais aussi conscience du cinéma, car si les films construisent un regard, ils n'en convoquent pas moins le cinéma. «Ces entrées de cinéma (qui ne sont ni nommées, ni même 
visibles) déterminent la structure du film, son montage, pour ne plus laisser que des traces, des "sensations de cinéma" /32 ».

Carnet de route, collecte vagabonde, errance, nomadisme, l'essai est une forme qui parcourt le monde dans une volonté de s'éprouver dans le paysage, mais aussi une forme qui se cherche, qui s'invente dans l'essai en traversant des territoires comme autant d'expériences d'images. Le programme de la maison de production Anna Sanders Film s'énonce ainsi : «ce projet s'est voulu un moyen d'inventer - par le film - des moments de paysage. Anna Sanders rêve de faire partager au spectateur sa liberté dans la perception des durées et des temporalités/33. " Partant d'une expérience du paysage qui mène à l'invention d'une forme, les films-essais produits par Anna Sanders semblent donc motivés par une volonté de faire naître un paysage à l'image.

Le film Le Pont du trieur de Philippe Parreno et Charles de Meaux (1999) avait comme intention annoncée une question: un pays dont on ne connaît pas d'images appartient-il encore à l'histoire? Le film serait une traversée du paysage, de territoires à peine ouverts aux étrangers, précisément la région reculée du Pamir au Tadjikistan. C'est pourquoi la forme du film découle du projet. Comme on n'a pas d'images du Pamir, le film commence par un écran blanc, puis un studio d'enregistrement et seulement ensuite des images de paysages. Réalisé à la façon d'un road movie, mais réduit à sa plus simple expression, «il s'agit de prendre la mesure de l'espace, du territoire, et de sa vacance /34 ", annonce Charles de Meaux. Un film qui raconte un pays sans images devient vite une forme qui teste son propre projet: «Faire naître le Pamir à la vérité des images»; une forme qui se révèle dans l'image, un film qui s'éprouve, en quelque sorte.

Marine Hugonnier, elle aussi parcourt le monde avec sa caméra, avec pour objectif de révéler le paysage comme forme culturelle codifiée. Dans Ariana (2003), l'équipe de tournage se trouve dans l'impossibilité de filmer la vallée du Panshir en Afghanistan, ce qui l'amène à s'interroger sur les spécificités du paysage et le sens du panorama. The Last Tour (2004) nous emmène en ballon au-dessus des montagnes. En écho au temps des attractions du siècle passé, panoramas peints ou aménagements de sites naturels, ce voyage en ballon interroge les différentes réglementations et codifications du paysage. Travelling Amazonia (2006) est une traversée de la forêt amazonienne. Toutefois, si l'élaboration du film repose sur le tracé de la ligne de tournage, la construction d'une Dolly, la mise en place d'un travelling, la nature est néanmoins inhérente au processus du film. Effectivement, comme en résonance à l'Histoire, - ici précisément le grand projet de développement de la Transamazonienne à l'époque
/32 Stéphanie Moisdon, Pointligneplan, op. cit, p. 103.

/33 Propos de la bande annonce de Anna Sanders Films.

/34 François Bovier et Cédric Fluckiger, «À propos d'Anna Sanders: entretien avec Charles de Meaux", Décadrages, op. cit., p. 84 . 
/35 Jean-Pierre Rehm, «Ariane Michel», texte de présentation de l'invitation de la séance Pointligneplan. [en ligne] www.pointligneplan.com/ les-hommes-texte

/36 Murielle Gagnebin en écho à Godard, «L'Essai est une forme qui pense», dans «L'inconscient à l'essai », op. cit., p. 13. des dictatures des années 1970, qui devait permettre de transporter et commercialiser les ressources de la forêt - ce projet doit puiser ses ressources dans le paysage à traverser. Bois, fer et caoutchouc seront donc les matériaux de base servant à l'élaboration du travelling. Par cette réflexion sur l'opération cinéma, Travelling Amazonia met en présence les enjeux esthétiques, économiques et politiques à l'échelle d'un film mais aussi d'un pays et de son Histoire.

De même, le travail d'Ariane Michel éprouve et révèle le paysage, mais dans la perspective plus particulière du monde animal. Sur la Terre (2004) suit deux phoques au Groenland. Les Yeux ronds (2004) sont ceux d'une chouette à Paris qui regarde l'activité de la place de la Concorde. Les Hommes (2006) montre le quotidien paisible des animaux, perturbé par un groupe de scientifiques qui débarquent pour une mission d'exploration. Ainsi, dans une radicalité assumée du documentaire animalier, elle n'hésite pas à retourner le processus du regard, ne partant plus de l'homme, du visiteur ou de l'explorateur, mais de la nature même. Procédant à des choix toujours plus affirmés, elle épuise les ressources cinématographiques et invente une forme singulière: pas de récit, pas d'histoire, pas de souffle, pas de parole, pas de trace de sa présence. En effet, le film semble même tourné à hauteur d'animal, voire à hauteur des pierres si l'on suit l'artiste qui confie se caler dans le monde des pierres. "C'est, plutôt que se prétendre pierre soi-même et poser ou creuser un point de vue, l'inventer. Le laisser pousser, à la manière d'un végétal, dans un espace intermédiaire. Intervalle, autrement dit, d'intercession. Un tel point de vue ne capture rien, ne se saisit de rien: il intercède pour ce qu'il contemple /35. ». Le dispositif mis en œuvre tente l'effacement du filmeur, qui ne semble plus que contribuer discrètement aux opérations techniques de captation des images et des sons. On se retrouve donc seuls face à l'image filmée et, comme cette image est celle du Monde, des animaux, de la Terre, on a l'impression que le film est comme une somme de la Nature, la somme de ses éléments, végétal, minéral et animal. Et quand l'image, très lentement, suivant le cours du bateau, passe du blanc de la glace à la couleur de la végétation discrète de la terre, on assiste à la révélation d'une image. Tout comme dans l'essai littéraire, ici l'artiste réinvente, intériorise, intimise, jusqu'à se découvrir une dimension existentielle.

\section{L'essai comme forme qui pense /36}

En 1948, Alexandre Astruc imagine un nouveau cinéma comme une "caméra stylo». "Le cinéma est en train tout simplement de devenir un moyen d'expression, ce qu'ont été tous les autres arts avant lui. 
[...] Un langage, c'est-à-dire une forme dans laquelle et par laquelle un artiste peut exprimer sa pensée, aussi abstraite soit-elle [...] aujourd'hui déjà un Descartes s'enfermerait dans sa chambre avec une caméra de $16 \mathrm{~mm}$ et de la pellicule et écrirait le Discours de la méthode en film car son Discours de la méthode serait tel aujourd'hui que seul le cinéma pourrait convenablement l'exprimer/37.». C'est ce que confirme à sa manière Marcel Ophuls lors de la sortie du documentaire Le Chagrin et la pitié (1971), lorsqu'il déclare que l'on a maintenant, grâce notamment à une technologie plus légère, la possibilité de travailler les idées et les paroles comme un écrivain /38. Caméra stylo ou prise de son direct, la parole est libérée, fluide comme une pensée à voix haute. Formes qui pensent, "c'est-à-dire des formes qui cheminent vers la parole /39 ", l'essai se donne voix et peut dès lors la donner à l'autre. S'emparant du film-essai, les artistes ouvrent des espaces de parole, mettent en place des dispositifs: entretiens, témoignages filmés, lieux de langage ou même quête d'une parole perdue.

Le film Intervista (1998) de Anri Sala a pour point de départ un événement mineur: la découverte lors d'un déménagement d'un film d'actualité qui relate un congrès de la jeunesse communiste en Albanie. Mais ce film, auquel il manque le son, n'est pas seulement un document d'Histoire. L'un des protagonistes, qui occupait alors un poste officiel, n'est autre que la mère de l'artiste. Aussi Sala va mener l'enquête afin d'exhumer un langage perdu à double titre, par la perte matérielle de la bande-son, mais aussi par le travail de la rhétorique politique. Il demande alors à des sourds-muets de déchiffrer les propos du film afin de restituer la parole aux images qu'il insèrera en sous-titres. Ce langage se révèle être celui de la propagande communiste de l'époque, langage officiel qu'il confronte de suite à la mémoire incrédule de sa mère. Pas de jugement à l'œuvre ici, ni même de volonté de reconstruction historique, mais un essai qui s'articule autour du personnel et du politique, et rend manifeste la friction entre langage et image, entre mémoire et Histoire.

Travaillant à nouveau sur le langage dans Nocturnes (1999), Sala confronte deux paroles à priori sans relation, celle d'un ex-soldat des Balkans et celle d'un amateur de poissons d'aquariums. Toutefois, le montage fait résonner les deux propos. Une petite leçon sur la nécessité d'une période d'adaptation d'un nouveau poisson au sein de cette communauté de prédateurs renvoie à ceux de la barbarie de la guerre. Autre écho, l'ex-soldat dit passer ses insomnies et cauchemars par une immersion dans les jeux vidéo, diluant ainsi sa mémoire dans des fictions virtuelles de guerres... En tension entre réalité et fiction, le film témoigne d'un réel qui a déjà basculé.
/ 37 Alexandre Astruc, «Naissance d'une nouvelle avant-garde », L'Écran Français, n 144 , 30 mars 1948, publié dans Alexandre Astruc, Du stylo à la caméra... et de la caméra au stylo, Paris, L'Archipel, 1992, p. 325.

/38 Marcel Ophuls, «Presque en essayiste, de mélanger ce qu'étaient traditionnellement le roman et l'essai», Cinéma $71, \mathrm{n}^{\circ} 157$, juin 1971, p. 38.

/39 Jean-Luc Godard, Histoire(s) du cinéma (1989-1998). 
/40 «We could be heroes just for one day », Paris, ARC-MAMVP, déc. 2008/ mars 2009.

/41 Je veux voir, long-métrage avec Catherine Deneuve et Rabih Mroué, sorti en salles en décembre 2008.

/42 François Bégaudeau, «Olivier Zabat, cinéaste malgré lui », Les Cahiers du Cinéma, nov. 2005 , p. 33
Dans ce discours qui oscille entre Histoire, politique et actualité, citons les deux films de Hadjithomas et Joreige dernièrement montrés en installation /40 et intitulés du nom d'un camp de détenus au Liban, Khiam (2000-2007). Composé de deux séries de témoignages de six anciens détenus d'un camp, interrogés à huit ans d'intervalle, assis face à la caméra, le film fait le pari de la sobriété et le montage thématique révèle comment chacun s'arrange d'une situation subie. S'ils évoquent leur détention dans le premier film, le second fait part de points de vue plus complexes. Effectivement, entre les deux films, le camp a d'abord été transformé en Musée, puis détruit lors des assauts israéliens, pour être actuellement en cours de reconstruction en lieu de commémoration. Ainsi, six individus évoquent leur lieu de détention, s'expriment sur l'importance ou non de garder ou de montrer ce qui a été, sur la nécessité de penser l'inscription de la mémoire. Ils s'inquiètent qu'une reconstitution du camp n'en efface paradoxalement la mémoire. Khiam travaille l'imbrication complexe, le recouvrement de strates de mémoire et d'Histoire. D'ailleurs, dans Je veux voir (2008) /41, telle une allégorie sur l'effacement de la mémoire, les grues transportent et transforment en poussière les ruines de la guerre afin de les faire disparaître dans le paysage. Toutefois le film résiste en affirmant qu'il veut voir, qu'il veut montrer, qu'il veut mettre en mémoire malgré tout.

Dans Zona Oeste (1999), Olivier Zabat ne fait pas un documentaire sur les guérillas dans les favelas de Rio de Janeiro, mais il met en place des espaces cinématographiques distincts où chacun des groupes opposés s'affirme dans le langage et sa mise en scène (frontalité, ton, jeux, masques). Pas d'effet de réel ici, juste un impact d'une extrême violence, pour un film en triptyque où chacun affirme son rôle face à la caméra. «Film stupéfiant. Film à l'identité double. S'agit-il encore d'un film d'art, s'agit-il déjà d'un documentaire /42 ? » Zabat travaille par accumulation de séquences, de reprises, qui, dispersées au sein de chaque film, se croisent tout au long de sa filmographie, de Miguel et les mines (2002) à 1/3 des yeux (2004). Artiste passé pleinement à la réalisation, chaque film tisse ainsi un réseau en fragile tension. Cela produit un cinéma à l'équilibre précaire, à l'image de ses sujets.

Les films de Clarisse Hahn, dans une volonté de «dresser des portraits", se rapprochent d'individus formant des groupes ou des collectivités qui ne revendiquent pas tant leur individualité que leur attachement à une codification collective. «Je m'interroge sur la manière dont l'identité résiste, ou bien se fond à l'intérieur d'une communauté», dit la réalisatrice. Usant du procédé qu'offre le témoignage filmé, mais accentué au profit d'une certaine proximité, 
l'attention portée au corps le révèle comme corps social dans la manière dont il se lie ou se délie de son appartenance à un groupe, à une identité. Dans Les Protestants (2005), le milieu montré est celui de l'artiste et pourtant, loin du film familial, voire de vacances, on y sent une résistance des individus au jeu de la caméra, comme si chacun refusait l'isolement des entretiens individuels, préférant se présenter dans son appartenance au groupe. Est-ce bien une tante qui lui demande si elle n'a jamais éprouvé la fierté d'être soumise à son époux, ce à quoi l'auteur ne sait répondre tant s'est creusée la faille d'incompréhension, de différence. Le lien familial s'est rompu... Et le corps filmé semble alors se rétracter dans l'image. S'attardant sur l'image du corps, son apparence, son appartenance, elle guette les moments où le lien lâche. En témoigne cet autre film Hôpital (1999) où l'on assiste à une mécanique des gestes opérés sur les patients du service de gériatrie qui semble, au profit d'une hygiène nécessaire, les déposséder de leurs propres corps.

Florence Lazar travaille quant à elle sur un sujet complexe, l'après conflit en ex-Yougoslavie. Pour son film PRVI DEO (réalisé avec Raphaël Grisey, 2006), elle prend pour point de départ le premier procès pour crime de guerre en Serbie. Faisant parler les familles des victimes dans l'hôtel proche, elle libère une parole qui évoque, remémore et interroge ce qui échappe à l'entendement (à celui des victimes comme au nôtre). Ailleurs, c'est un long plan séquence de nuit dans une voiture où la conductrice hors champ nous mène sur les lieux du massacre de Vukovar. Mais cette voix sans image a beau nous conduire, nous montrer et nous raconter, nous restons face à des signes invisibles, indéchiffrables par notre regard. Le film bute alors sur l'impossibilité de la représentation. Références à la peinture d'Histoire (picturalité du paysage, des sujets que l'on retrouve aussi dans ses photographies), création de lieux de parole /43, images non didactiques, Lazar met en place les conditions d'une pensée politique qui interroge: comment les identités se construisent sur des points de vue, combien la réalité peut être multiple et enfin comment l'actualité répond à l'Histoire?

Affirmant la singularité de la pratique de la pensée, l'essai est avant tout une prise de parole. Cette pensée, cette parole, l'auteur l'assume et la fait découvrir en passant par le texte ou l'image. «Dans un film documentaire dit de création ou d'auteur, la voix est celle des images/44. » Parole temporelle voire précaire, si l'essai se tourne vers l'histoire, c'est par articulation avec le présent, mais un présent qui manifeste sa subjectivité. S'énonçant comme il pense, il ne cautionne que lui-même. L'essai est une parole qui se tient devant les choses et devant le temps.
/43 Voir aussi le film Les Femmes en noir (2002), collection MNAM, Centre Georges Pompidou. «m.m.m. (moments. mondes.modernités.)", dans Pointligneplan, op. cit. p. 106.

/44 François Niney, L'Épreuve du réel à l'écran, op.cit., p. 238. 
/45 Voir à ce propos le texte de Marielle Macé, «L’Essai littéraire, devant le temps», Cahiers de Narratologie, $\mathrm{n}^{\circ} 14$, février 2008 , [en ligne ] http:// revel.unice.fr/cnarra/ document.html id $=499$

/46 Alexandre Astruc, «Naissance d'une nouvelle avant-garde», op. cit., p. 325-326

\section{Art et cinéma, l'essai comme langage}

L'essai au cinéma - qui s'affirme dans sa singularité et repousse constamment les limites de sa possible définition - trouve toutefois son identité dans ses usages. Forme existentielle se tenant devant le temps, il se manifeste avec la force d'un Je rendu disponible. Forme qui se risque, il initie un cinéma où l'on se trouve en même temps que l'on s'invente, où l'on trouve en même temps que l'on invente /45. Se permettant tous les écarts entre le Je et les autres, entre objectivité et subjectivité, entre réel et non réel, il chemine aisément du documentaire à la fiction. Forme qui pense, prise de parole et réflexion par l'image, l'essai cinématographique ne cesse d'élargir son territoire et revêt toutes les formes possibles d'un langage annoncé par Astruc: «des films écrits sur n'importe quel sujet, de n'importe quelle forme, aussi bien critique littéraire, roman, qu'essai sur les mathématiques, histoire, vulgarisation, etc. Dès lors il n'est déjà plus permis de parler d'un cinéma. Il y aura des cinémas comme il y a aujourd'hui des littératures, car le cinéma comme la littérature, avant d'être un art particulier, est un langage qui peut exprimer n'importe quel secteur de la pensée /46. ». Et cette éclosion des cinémas trouve aussi son écho dans le champ de l'art contemporain. Tout à la fois et tour à tour, le film-essai se fait forme entre documentaire et fiction, forme qui se risque, carnet de route, et enfin, forme qui pense, quatre oscillations qui donnent la mesure aux propositions cinématographiques des artistes contemporains. Des films réalisés par des artistes qui affirmaient leur réticence vis-à-vis des définitions de genre (documentaire et fiction), tout en s'affranchissant du cinéma expérimental, nous nous trouvons aujourd'hui en face d'un territoire que seul le genre de l'essai peut réunir et rassembler cinématographiquement. Car, fort de son double héritage littéraire et cinématographique, le genre de l'essai est une forme ouverte à la multiplicité des cinémas, un langage ouvert à la multiplicité de la pensée. Les artistes, en adoptant ce nouveau genre, s'expriment ainsi pleinement dans le cinéma.

\section{Véronique Terrier Hermann}

